\title{
Temperatures in a runaway greenhouse on the evolving Venus: implications for water loss
}

\author{
Andrew J. Watson ${ }^{1}$, T.M. Donahue ${ }^{2}$ and W.R. Kuhn ${ }^{2}$ \\ ' Marine Biological Association of the U.K., The Laboratory, Citadel Hill, Plymouth PLI 2PB (U.K.) \\ 2 Department of Atmosphere and Ocean Science, University of Michigan, Ann Arbor, MI 48109 (U.S.A.)
}

Received January 4, 1983

Revised version accepted January 16, 1984.

\begin{abstract}
Recent work has established that Venus once had at least 100 times its present complement of outgassed water. An original complement of water comparable to the earth's is not inconsistent with our present knowledge (and is worthy of consideration since it involves no special assumptions concerning the differences in origin of the two planets). We use a one-dimensional calculation to investigate temperatures in the "runaway greenhouse" which may have ensued if Venus once had more than a few percent of the earth's water complement. We find that the atmosphere must exhibit an unusual structure, with condensation and presumably cloud formation taking place at high altitudes, while deep in the atmosphere the gas is strongly unsaturated with respect to water vapour. The necessity of including clouds introduces considerable uncertainty into the calculation of surface temperatures. However, for certain reasonable values of the cloud parameters, very high temperatures can be sustained, approaching the temperature of the basalt solidus. We speculate that such high temperatures may have promoted water-rock reactions, releasing free hydrogen and "burying" oxygen. A plastic or molten surface could have promoted rapid exposure of fresh rock, significantly easing the problem of disposing of the oxygen released by the dissociation of water.
\end{abstract}

\section{Introduction}

The recent measurement of the deuterium/ hydrogen ratio in the Venus atmosphere [1], coupled with improvements in our understanding of hydrogen escape processes [2,3], makes it nearly certain that Venus once had at least 100 times its present-day abundances of water. The assumption of an "earth-like" initial complement (say, $10^{3}$ to $10^{4}$ times the present Cytherean abundance of water), is consistent with our present knowledge and is worthy of serious consideration since it is not predicated upon any particular theory for the origin of the planets. If Venus did once have abundant water, it is likely that the event which precipitated its loss was a "runaway greenhouse" in which the oceans boiled into the atmosphere. Subsequently the water dissociated through the absorption of EUV in the upper atmosphere. The hydrogen escaped from the planet while the oxygen reacted with the rock exposed by tectonic processes. So at least goes the theory expounded by Walker [5], building on the work of a number of earlier authors [6,7]. One of the remaining problems of this long-standing hypothesis is that it requires extremely active vulcanism to expose enough rock to react with all the oxygen $[4,5]$. In this paper we examine some aspects of the temperature structure of a runaway greenhouse using one-dimensional radiative transfer techniques. We find that there generally is a region high in the atmosphere where condensation and cloud formation can occur: clouds must therefore by included in any model and this introduces a number of uncertainties in the calculation of temperatures. Under reasonable assumptions concerning the clouds, temperatures deep in the atmosphere are high enough to produce a plastic or even molten 
surface, which may significantly ease the problem of explaining the loss of oxygen.

The runaway greenhouse was first described by Ingersoll [6]. It may be defined as a condition in which bodies of liquid water cannot condense at the surface; instead, all the water must reside in the atmosphere. Ingersoll argued that. if a planet has a stable ocean, the lower atmosphere must be close to saturation. This must be so because only then can water evaporating from the surface be returned to it by condensation and precipitation. However, there is a limit to the amount of thermal radiation that a saturated atmosphere is capable of losing to space [7]. If the planet is too close to the sun, it may absorb more solar energy than it can radiate, causing the surface temperature to rise. Flux balance can only be restored when the oceans have evaporated entirely and the atmosphere can become unsaturated. Pollack [7] investigated the conditions leading up to runaway. He concluded that, provided the cloud cover was substantially less than $100 \%$, runaway could have occurred on Venus. Our purpose here is to provide a first-order description of the atmosphere after runaway has taken place. We assume the atmosphere contains 90 bar of $\mathrm{CO}_{2}$ and, in addition, between 10 and 90 bar of water vapour. For comparison, if an entire earth-ocean of water was originally present on Venus, the partial pressure of $\mathrm{H}_{2} \mathrm{O}$ at the surface would be about 300 bar.

The next two sections of this paper describe the techniques and results of respectively upper and lower atmosphere calculations for the temperature structure. We then discuss some of the uncertainties in the surface temperatures obtained from the model. The final section discusses the implications of these calculations in the context of the evolution of the Venus atmosphere.

\section{Upper atmosphere}

Fig. 1 shows radiative equilibrium pressuretemperature profiles for the upper portion of an atmosphere containing 90 bar each of $\mathrm{CO}_{2}$ and $\mathrm{H}_{2} \mathrm{O}$. The profiles (and all the results quoted here) are appropriate to globally averaged conditions with the solar constant taking its present-day value

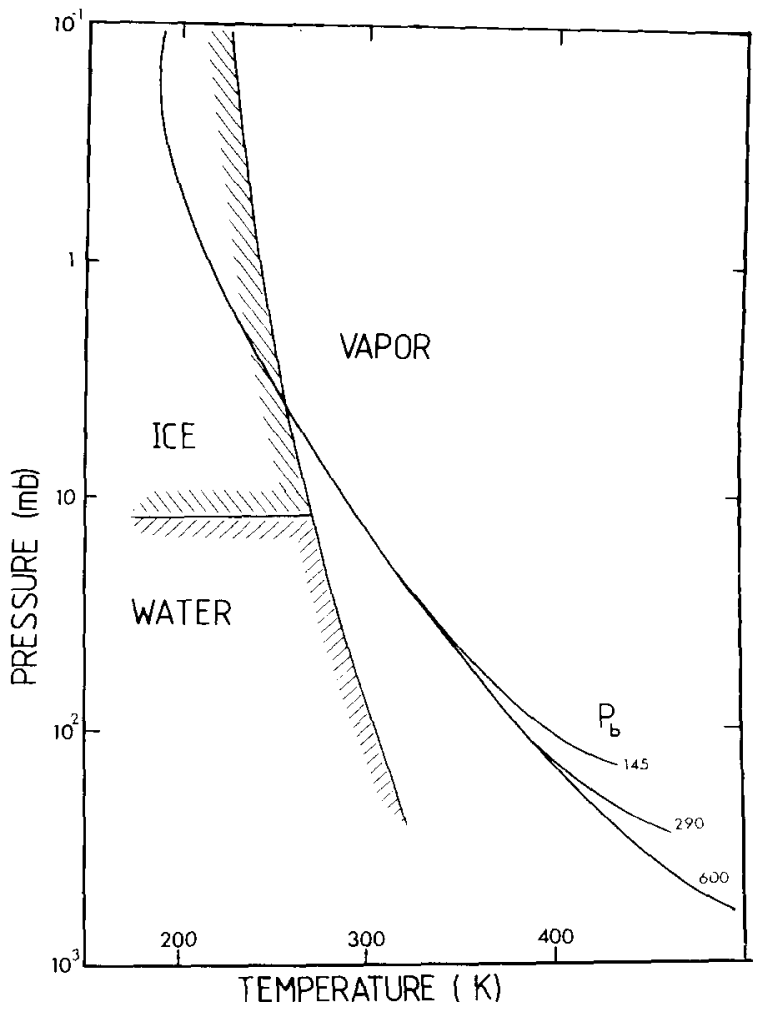

Fig. 1. Radiative equilibrium for a $50 \% \mathrm{H}_{2} \mathrm{O}, 50 \% \mathrm{CO}_{2}$ atmosphere for three values of the pressure at the lower boundary. Condensation processes are ignored. The phase transitions of the water are also shown. Temperatures near the top of the atmosphere are too low for water to remain in the vapor phase. The distortion of the profiles due to the artificial lower boundary is a local effect provided $P_{b}$, the pressure in mbar at which the boundary is situated, is large enough.

at the orbit of Venus. The computational techniques were adapted from well-tested programs used in previous applications $[8,9]$. Transmission functions were calculated from AFCRL data [10] using the quasi-random band model of Wyat et al. [11]. In the $8-12 \mu \mathrm{m}$ and $4 \mu \mathrm{m}$ windows, the "e" type absorption of water vapour was modelled with data from Bignell [12]. The coefficient of e-type absorption, $K_{e}$, was assumed to vary as:

$K_{\mathrm{e}} \propto \exp \left(E_{\mathrm{a}} / R T\right)$

where $E_{\mathrm{a}}$ is $5 \mathrm{kcal} / \mathrm{mol}$, the activation energy of the hydrogen bond, $R$ is the gas constant and $T$ is temperature. An artificial lower boundary was used in performing the computations of Fig. 1, arbitrarily fixed at a level of $P_{\mathrm{h}}$ mbar. $P_{\mathrm{h}}$ was chosen 
large enough that all the thermal radiation escaping to space was emitted from the region above $P_{\mathrm{b}}$. The boundary was modelled as a black body in the infrared and as a constant albedo surface in the visible. The black-body assumption tended to distort temperatures near the boundary, but this was a localized effect provided $P_{\mathrm{b}}$ was sufficiently large, as may be seen in the figure. The visible albedo was assumed to be due to Rayleigh scattering from the lower atmosphere, and was calculated by the method of Pollack [13].

The profiles of Fig. 1 are supersaturated with water at high altitudes. One would therefore expect that ice clouds could form, and that lapse rates greater than the moist adiabatic would be unstable to convection. Accordingly, we modified the calculation to include a model cloud. The cloud was considered to have negligible vertical

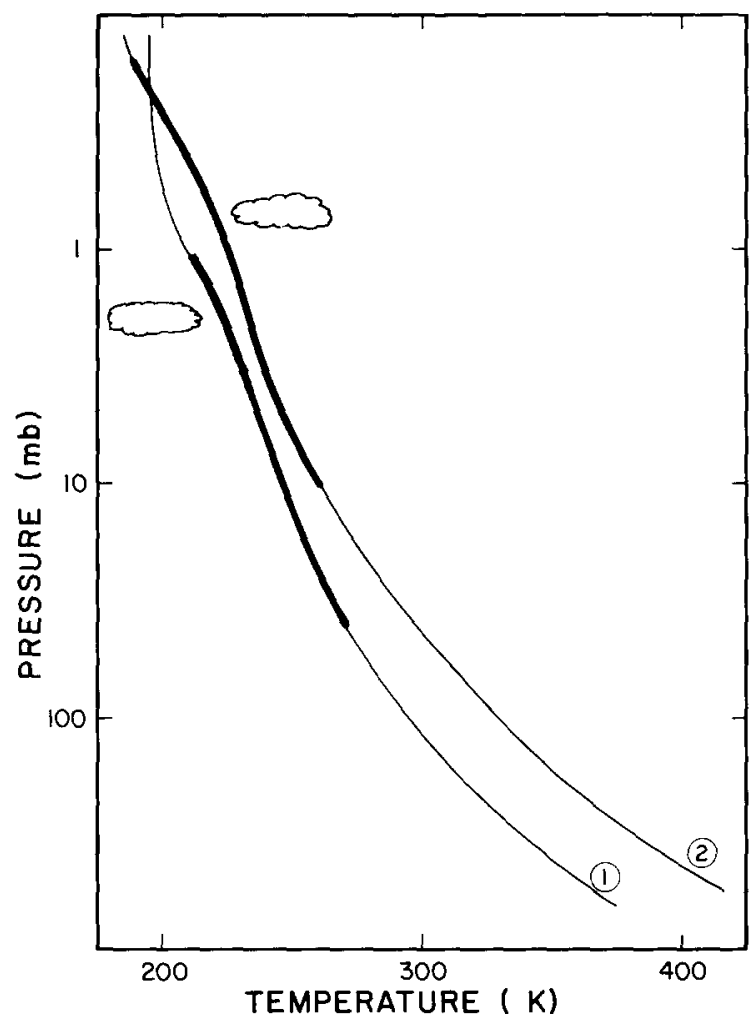

Fig. 2. Higher atmosphere radiative-convective pressure-temperature profiles. The heavier lines show regions where the curves follow a moist adiabat. Profiles are for $10 \% \mathrm{H}_{2} \mathrm{O}$ in the atmosphere. $I$ : cloud albedo $=90 \% ; 2:$ cloud albedo $=55 \%$. The mean planetary albedos are respectively $46 \%$ and $63 \%$. extent, to be black to infrared and to have a fixed albedo in the visible, and was placed in the saturated region. Where the new calculation predicted saturation the lapse rate was constrained to be no greater than the moist adiabatic, calculated by the method outlined by Ingersoll [6]. Fig. 2 shows the results for cases where the cloud albedo was $55 \%$ and $90 \%$ and the cloud covered $50 \%$ of the planet. When flux balance was achieved, the atmosphere exhibited a structure in some ways the inverse of the earth's; a region in moist-convective equilibrium overlaid an unsaturated region in radiative equilibrium. By contrast, on earth an extensive zone in radiative equilibrium (the stratosphere) overlies the troposphere which is in moist convection. As predicted by Ingersoll, the true stratosphere in the runaway greenhouse begins only at very low pressures.

It is of interest to follow the response of the model to changes in the amount of absorbed radiation. As previously discussed, in a runaway greenhouse the saturated portion of the atmosphere is not able to deliver the required upward flux to balance the absorbed solar radiation. It follows that, at flux balance, sufficient radiation from the unsaturated region must escape to make up the difference. This requirement constrains the extent of the moist-convective region, which must be transparent enough to allow radiation from below to escape. As one lowers the absorbed radiation, (for example by "turning down" the solar constant), the saturated region extends to progressively greater depths. At some point it becomes so optically thick that it entirely determines the outgoing flux. Condensation may then proceed downwards to the surface and the runaway greenhouse collapses.

\section{Lower atmosphere}

Using pressures and temperatures from the upper atmosphere calculation as a starting point, we then applied Pollack's [13] formulation to find the temperature structure down to the surface. The lower atmosphere calculation was patched to that for the upper atmosphere at a point well above $P_{\mathrm{b}}$ so as to eliminate errors due to the artificial 


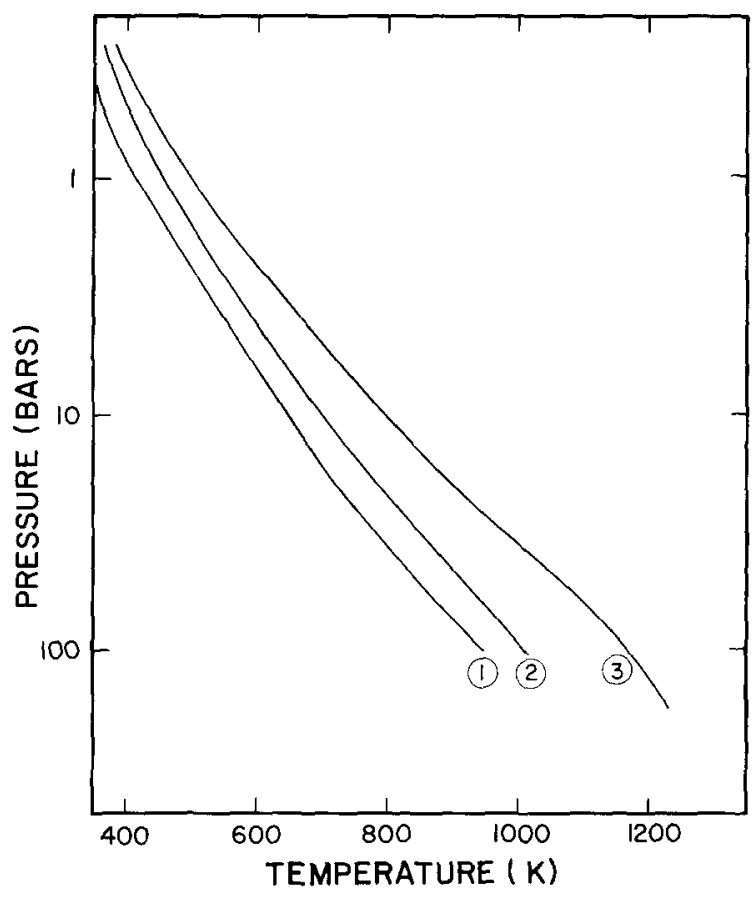

Fig. 3. Temperatures in the lower atmosphere. $\mathrm{I}: 10$ bar $\mathrm{H}_{2} \mathrm{O}$, cloud albedo $=90 \% ; 2: 10$ bar $\mathrm{H}_{2} \mathrm{O}$, cloud albedo $=55 \% ; 3: 90$ bar $\mathrm{H}_{2} \mathrm{O}$, cloud albedo $=90 \%$.

boundary. The two methods appeared to be consistent in that they gave approximately the same temperature structures near the point where they were joined.

Some examples of lower atmosphere solutions are given in Fig. 3. Because of the very high temperatures reached at depth in some of the calculations, it was necessary to extend Pollack's data for infrared opacities to shorter wavelengths. We obtained the mass path and pressure dependence of opacity, in thirteen wavelength intervals between 2.1 and $0.9 \mu \mathrm{m}$ using the same data source and methods as for the upper atmosphere. This procedure involved some extrapolation, as well as the neglect of temperature dependence and pressure-induced absorption effects: our results for the lower atmosphere must therefore be regarded as estimates only. Water vapour was the dominant source of opacity at nearly all wavelengths. Deep in the atmosphere our calculations indicated that the dominant window was at 1-1.05 $\mu \mathrm{m}$ with relatively transparent regions near 1.22 $\mu \mathrm{m}$ and $1.6 \mu \mathrm{m}$. However, recent work suggests that $\mathrm{CO}_{2}$ has substantial pressure-induced absorption near $1 \mu \mathrm{m}$ so we may have underestimated the opacity in this window and hence also the temperatures in the lowermost atmosphere. Temperatures followed a (dry) adiabat through most of the deep atmosphere, but a stable region was in some cases encountered near the surface. where the solar flux became too weak to sustain the rapidly increasing temperatures. Solar radiation penetrating to the ground was mostly confined to wavelengths less than $0.55 \mu \mathrm{m}$ and was strongly attenuated by Rayleigh scattering. The net flux at the surface was typically only $0.1-1 \%$ of that at the top.

\section{Surface temperatures and sources of uncertainty in the model}

Table 1 shows surface temperatures as a function of the amount of water vapour in the atmosphere and the albedo chosen for the clouds. Cloud cover was fixed at $50 \%$. The surface temperatures are very high indeed and this may have important consequences for the evolution of the planet, as discussed below. However, before proceeding to discuss the implications we should clearly state the limitations of our calculations. Considerable uncertainty is introduced by the necessity to include clouds in the model. We have not attempted to predict cloud properties in a self-consistent calculation, but have instead regarded cloud cover, albedo, and height as fixed parameters. In reality these properties would be determined by the meteorology of the runaway greenhouse, but we are dealing with an atmosphere so unlike any on

TABLE 1

Surface temperatures as a function of the total amount of water vapor in the atmosphere

\begin{tabular}{llc}
\hline Water vapor (bar) & \multicolumn{2}{l}{ Surface temperature $(\mathrm{K})$} \\
\cline { 2 - 2 } & cloud albedo $=55 \%$ & cloud albedo $=90 \%$ \\
\hline 10 & 1010 & 950 \\
20 & 1090 & 1030 \\
50 & 1200 & 1150 \\
90 & 1270 & 1230 \\
\hline
\end{tabular}


the modern-day planets that there are as yet few constraints on its physical properties. Our calculations show only that some condensation must occur, and that for some reasonable values of the cloud parameters very high surface temperatures are sustained. These temperatures are moderately sensitive to changes in the cloud parameters. For example, for the case of 90 bars $\mathrm{H}_{2} \mathrm{O}$, increasing the cover to $60 \%$ decreased the surface temperature by $80 \mathrm{~K}$ when the cloud albedo was $90 \%$, and by $40 \mathrm{~K}$ when it was $55 \%$. Highest surface temperatures were usually generated when the clouds were left out of the calculation.

Another source of uncertainty is the value of the solar constant. It is now thought quite likely that the output of the sun has increased, by perhaps $30 \%$., since the solar system was formed 4.5 billion years ago. In that case the model solar flux should be decreased from its present value by an amount which depends on the time at which the runaway is deemed to have taken place. This leads to lower temperatures; indeed, for the high cloud albedo cases a $30 \%$ reduction in solar luminosity was sufficient to induce the collapse of the runaway greenhouse, implying that surface temperatures would be less than the critical temperature of water [5], $647 \mathrm{~K}$. If the sun has really increased its output substantially, this result may suggest a relatively late data for the Venusian runaway greenhouse.

\section{Implications}

The temperatures in Table 1 are generally rather lower than the solidus of dry basalts (about 1230 K). However, they are high enough for the surface rocks to be highly plastic, promoting active vulcanism. Furthermore, at temperatures in excess of $1000 \mathrm{~K}$ the water would react quite rapidly with the rock by reactions such as:

$3 \mathrm{FeO}+\mathrm{H}_{2} \mathrm{O}=\mathrm{Fe}_{3} \mathrm{O}_{4}+\mathrm{H}_{2}$

Oxygen would be "buried" and the released hydrogen would be available for escape to space.

Models for the thermal history of Venus suggest that, not far below the surface, the planetary interior is (and may always have been) in partial melt
[14]. A hot and plastic surface might therefore allow the frequent extrusion of molten material, resulting in a large mass of rock being brought into contact with the atmosphere. This would provide a rapid sink for oxygen at the surface. The rate of dissipation of the greenhouse would have been controlled by the rate at which rock was brought into the reaction zone, or, if this was sufficiently fast, by the rate at which hydrogen could escape the planet. In the latter case the lifetime of the runaway greenhouse may have been only a few times $10^{8}$ years.

The picture is complicated slightly if we consider the possibility that the solar luminosity has been increasing slowly with time, and that a resulting increase in absorbed radiation triggered the runaway some time after the formation of the planet. Immediately after the event the sun was only just hot enough to sustain runaway and it is possible that surface temperatures were not high enough to induce rapid surface turnover. Initially then, oxygen released by upper atmosphere photodissociation would build up in the atmosphere while hydrogen would escape to space. If we suppose this process to continue indefinitely, all the water in the atmosphere would in time be replaced by some tens or hundreds of bars of oxygen [5]. This is not a very appealing scenario for it is hard to see how such a super-oxidizing atmosphere could subsequently evolve to the present redox-neutral condition with only ordinary tectonics to provide the oxygen sink. It also seems an unlikely hypothesis, from both a thermodynamic and a kinetic point of view, for as the oxygen began to accumulate it would certainly have tended to suppress the hydrogen concentration. The hydrogen escape rate would thereby be reduced until, at the steady state, it would be constrained to equal the surface oxygen sink. We think it most likely therefore that the water vapour atmosphere would dissipate only slowly until such time as the surface temperature, responding to the increasing brightness of the sun, became high enough to greatly accelerate the rate of rock extrusion.

To conclude, we suggest that high temperatures supported by a runaway greenhouse may have been important in the evolution of Venus, promoting the dissociation of water by reaction with 
surface rocks, and allowing the oxygen originally contained in the water to be thoroughly absorbed by the planet. If Venus once had the same water complement as the earth, there would initially have been some 300 bar of water in the atmosphere, most of which would have been rapidly destroyed by water-rock reactions with the evolved hydrogen escaping the planet in a copious hydrodynamic flow.

As the greenhouse dissipated, the surface became rigid and water-rock interactions would have been inhibited once the water vapour pressure dropped to about 20 bar. One is left with a remnant of water on the planet, the disappearance of which must be explained by other means. The relatively slow mechanisms originally proposed by Walker are probably adequate to dispose of this remnant. That is, the oxygen sink is provided by conventional volcanic processes, with upper atmosphere photodissociation splitting the water into its component elements.

\section{Acknowledgements}

We thank J.C.G. Walker for much encouragement and useful discussion on this topic. This research was supported by grant NAS-9126 from NASA.

\section{References}

1 T.M. Donahue, J.H. Hoffmann, R.R. Hodges, and A.J. Watson, Venus was wet: a measurement of the ratio of deuterium to hydrogen, Science 216, 630-633, 1982.
2 A.J. Watson, T.M. Donahue and J.C.G. Walker, The dynamics of a rapidly escaping atmosphere, Icarus 48 , $150-166,1981$.

3 M.G. McElroy, M.J. Prather, and J.M. Rodriguez, Escape of hydrogen from Venus, Science 215, 1614-1615. 1982.

4 J.S. Lewis and F.A. Kreimendahl, Oxidation state of the crust and atmosphere of Venus in the light of the Pioneer Venus results, Icarus 42, 330, 1980.

5 J.C.G. Walker, Evolution of the atmosphere of Venus, J. Atmos. Sci. 32, 1248-1256, 1975.

6 A.P. Ingersoll, The runaway greenhouse: a history of water on Venus, J. Atmos. Sci. 26, 1191-1198, 1969.

7 J.B. Pollack, A non grey calculation of the runaway greenhouse: implications for Venus' past and present, Icarus 14, 295-306, 1971.

8 W.R. Kuhn, S.K. Atreya and S.E. Postawko, Influence of ozone on Martian atmospheric temperature, J. Geophys. Res. 84, 8341-8342, 1979.

9 J.R. Hummell and W.R. Kuhn, Comparison of radiativeconvective models with constant and pressure-dependent lapse rates, Tellus 33, 254-261, 1981.

10 T.A. Mcclatchey, W.S. Benedict, S.A. Clough. D.E. Burch, R.F. Calfee, K. Fox, J.S. Rothman and J.S. Garing, AFCRL atmospheric absorption line parameters compilation. Environ. Research Pap. 34, Air Force Cambridge Research Laboratory, Hanscom Air Force Base. Bedford. Ma., 1973.

11 P.S. Wyatt, V.R. Stull, and G.M. Plass. The infra-red absorption of water vapour. Infra-red studies Final Rep. 2, SSD-TDR-62-127 Aeronutronic Division, Ford Motor Co., 1962.

12 K.J. Bignell, The water vapour infra-red continuum, Q. J. R. Meteorol. Soc. 96, 390-403, 1970.

13 J.B. Pollack, A non-grey $\mathrm{CO}_{2}-\mathrm{H}_{2} \mathrm{O}$ greenhouse model of Venus, Icarus 10, 314-341, 1969.

14 M.N. Toksöz, A.T. Hsui and D.H. Johnston, Thermal evolutions of the terrestrial planets, Planets 18, 281-320, 1978. 